\section{LA BIOTECNOLOGÍA EN SANIDAD ANIMAL}

Mạ José Rodríguez García

Ingenasa (Inmunología y Genética Aplicada S.A.)

mjrodriguez@ingenasa.com

Ana Ranz Casares

Ingenasa (Inmunología y Genética Aplicada S.A.)

aranz@ingenasa.com

Belén Barreiro Morán

Ingenasa (Inmunología y Genética Aplicada S.A.)

bbarreiro@ingenasa.com

Jacques Delbecque Peña

Ingenasa (Inmunología y Genética Aplicada S.A.)

jdelbecque@ingenasa.com

Antonio Sanz Fernández

Ingenasa (Inmunología y Genética Aplicada S.A.)

Paloma Rueda Pérez

Ingenasa (Inmunología y Genética Aplicada S.A.)

prueda@ingenasa.com

\section{ANIMAL HEALTH AND BIOTECHNOLOGY}

Cómo citar este artículo/Citation: Rodríguez García, M. J.; Ranz Casares, A.; Barreiro Morán, B.; Delbecque Peña, J.; Sanz Fernández, A. y Rueda Pérez, P. (2014). "La biotecnología en sanidad animal". Arbor, 190 (768): a154. doi: http://dx.doi. org/10.3989/arbor.2014.768n4008

Recibido: 9 junio 2014. Aceptado: 14 julio 2014.

RESUMEN: El crecimiento de la población mundial en los próximos años, provocará un incremento en la demanda de alimentos. Como consecuencia, el número de animales de producción sufrirá un aumento importante y la Sanidad Animal constituirá un elemento crítico a la hora de tratar y prevenir las enfermedades, garantizando el abastecimiento y la seguridad. La biotecnología contribuye de múltiples formas al desarrollo de la Sanidad Animal. Fundamentalmente, aporta herramientas que ayudan al control y erradicación de las enfermedades. El desarrollo de la ingeniería genética en las últimas décadas ha favorecido el avance de la industria biotecnológica aplicada a la sanidad humana y animal. Existen un gran número de ensayos de diagnóstico basados en desarrollos biotecnológicos, así mismo, cada vez hay más vacunas recombinantes contra enfermedades animales. La prevención y control eficientes de las enfermedades dependerán de mecanismos de detección temprana, que faciliten la toma de decisiones y una respuesta rápida.

PALABRAS CLAVE: Vacunación;VLPs; diagnóstico; inmunoensayos; DIVA; ensayos múltiples; ensayos de campo; PCR.
Copyright: (C) 2014 CSIC. Este es un artículo de acceso abierto distribuido bajo los términos de la licencia Creative Commons Attribution-Non Commercial (by-nc) Spain 3.0.

ABSTRACT: The growth of the world population in forthcoming years will cause an increase in the demand of food. As a result, the number of working animals is set to rise significantly and Animal Health will become a vital issue when it comes to treating and preventing diseases, and guaranteeing and uninterrupted and safe food supply. Biotechnology contributes to the development of Animal Health in many ways. First and foremost, it provides tools that help control and eradicate diseases. The progress of genetic engineering in recent decades has underpinned the development of the biotechnology industry applied to human and animal health. There are a large number of diagnostic tests based on biotechnological developments, and there is also an increase in the use of recombinant vaccines against animal diseases. An effective prevention and control of diseases will depend on early detection mechanisms that facilitate decisionmaking and enable a rapid response.

KEYWORDS: Vaccination; VLPs; diagnostic; immunoassays; DIVA; multiplex assays; field tests; PCR. 


\section{INTRODUCCIÓN}

Según un estudio reciente de la Organización de las Naciones Unidas, la población mundial llegará a los 9.600 millones en el año 2050. A comienzos del próximo siglo, el número de personas podría superar los 16.600 millones. La población aumentará de manera espectacular en las regiones más pobres del mundo, lo que provocará importantes problemas relacionados con la necesidad de agua y alimentos. Se calcula que la demanda mundial de proteínas animales habrá aumentado un $50 \%$ de aquí a 2030 , en parte debido a la emergencia de las clases medias en los países en desarrollo y a sus nuevos hábitos de consumo. Habrá un incremento importante en los censos de animales de producción y como consecuencia la Sanidad Animal tendrá cada vez más importancia.

Hoy en día, la Sanidad Animal, la Salud Pública, el Medio Ambiente, así como la Biomedicina, Producción Animal y Seguridad Alimentaria están íntimamente relacionadas. Así nace la actual iniciativa "One World, One Health", cuya intención es la de aglutinar el conocimiento y la investigación biológica, a todos los niveles (medicina humana, veterinaria y ciencias ambientales), a fin de conseguir mejorar la VIDA de todas las especies que compartimos el planeta. Trabajando juntos, se puede lograr más para mejorar la salud en todo el mundo y la profesión veterinaria tiene la responsabilidad de asumir un papel de liderazgo importante en ese esfuerzo (Rodríguez-Ferri, 2012).

Los últimos estudios epidemiológicos, muestran que el $60 \%$ de los patógenos humanos son de origen animal y que el $75 \%$ de las enfermedades animales emergentes pueden trasmitirse a humanos, es decir, son de carácter zoonótico.

La intensificación de la producción ganadera y agrícola durante el siglo XX, provocó la necesidad de la utilización masiva de agentes bactericidas y bacteriostáticos, especialmente antibióticos, a fin de mantener las condiciones sanitarias y los niveles productivos. Sin embargo, la tendencia actual trata de reducir el uso de antibióticos y se dirige hacia la prevención, control y erradicación de los procesos infecciosos en veterinaria, mediante estudios epidemiológicos, el diagnóstico y la vacunación, siendo los elementos clave para el control de la salud animal y en consecuencia de la salud humana.

Afortunadamente son cada vez más las herramientas disponibles para conseguir este objetivo, y en gran medida estas se basan en desarrollos biotecnológicos. Los avances en la Ingeniería genética en los últimos años han supuesto una verdadera revolución en el campo del seguimiento epidemiológico y de la fabricación de nuevos medicamentos, diagnósticos y vacunas. Si bien es cierto que suelen venir promovidos por su aplicación humana, en muchas ocasiones los logros en el campo de la veterinaria son pioneros por razones éticas.

Prescindiendo de los casos excepcionales más vanguardistas y revolucionarios, lo habitual es que por razones económicas, las tecnologías aplicadas a gran escala en los campos de la prevención veterinaria, sigan de cerca, pero un paso por detrás, los avances realizados en la medicina humana. A continuación se muestra de forma general cómo se aplican los procesos biotecnológicos en las áreas de la vacunación y el diagnóstico veterinarios.

\section{LAS VACUNAS EN VETERINARIA}

Una vacuna, según la definición tradicional, es un microorganismo completo, atenuado o muerto, o una parte de él, que tiene la capacidad de inducir una respuesta inmune protectora y duradera, similar a la generada en una infección natural, pero sin peligro para el individuo vacunado. Desde que Edward Jenner descubrió la primera vacuna frente a la viruela en 1796, estas se han utilizado con éxito frente a numerosos microorganismos. La utilización de las vacunas en sanidad humana y veterinaria ha contribuido enormemente a uno de los mayores logros del siglo XX: el control de las enfermedades infecciosas. Las primeras aplicaciones en Sanidad Animal fueron frente al carbunco, el cólera aviar, el mal rojo porcino o la rabia.

La vacunación ha sido una herramienta de vital importancia para el control de las enfermedades víricas en humanos. En los países desarrollados, los gobiernos han establecido programas de vacunación que han permitido controlar e incluso erradicar algunas enfermedades tales como la viruela, sarampión, etc. Las estrategias seguidas para controlar las enfermedades víricas en animales son similares a las utilizadas en humanos, sin embargo, en este caso los Gobiernos no intervienen siempre de la misma forma y, consecuentemente, el control de la enfermedad es más difícil. Conviene destacar que los virus favorecen la entrada de otros patógenos oportunistas, por ejemplo bacterias, por lo que la vacunación tiene además un efecto positivo en la reducción del uso de antibióticos.

Las vacunas clásicas se basan en agentes infecciosos manipulados, procedentes de cultivos celulares. Pueden ser: 
Vacunas inactivadas: se emplea el agente infeccioso intacto tras eliminar su capacidad infectiva mediante tratamientos físicos (calor) o químicos (formaldehido, tiomerosal, etc.). Son vacunas seguras, pero no activan la respuesta inmune citotóxica, por lo que son menos protectoras y necesitan formularse con adyuvantes potentes. Ejemplos de este tipo de vacunas son las del virus de la influenza equina, que se utiliza desde los años 60, o bacterianas como Yersinia ruckeri y Aeromonas salmonicida para peces o Salmonella para aves y ganado porcino.

Vacunas atenuadas: el agente infeccioso se atenúa generalmente mediante pases sucesivos en cultivo, produciendo una infección leve, sin sintomatología clínica y con gran capacidad inmunogénica. Sin embargo, tienen limitaciones importantes. Por una parte, la enfermedad puede ponerse de manifiesto, aunque levemente, como consecuencia de la vacunación y, por otra, en ocasiones el proceso de atenuación no es total y pueden llegar a revertir surgiendo variantes "salvajes" con capacidad de infectar y diseminarse. La utilización de una vacuna atenuada ha sido clave para lograr la erradicación de la peste bovina en 2011.

Posteriormente, surgieron las Vacunas de subunidades o de antígenos purificados: consisten en preparaciones más o menos puras de alguno de los componentes del virus. Generalmente son componentes proteicos, si bien pueden ser otro tipo de moléculas (por ej. azúcares). Se preparan mediante la extracción y separación de los componentes del agente infeccioso.

El desarrollo de la Biotecnología ha permitido diseñar nuevos tipos de vacunas más seguras y eficaces que reducen o eliminan los riesgos de las clásicas (Brun et al., 2011; Jackwood et al., 2008; Meeusen et al., 2007).

En general, estas nuevas vacunas presentan la gran ventaja de permitir la utilización de sistemas de diagnóstico diferencial (DIVA: Differentiate Infected from Vaccinated Animals), que pueden distinguir cuando un animal ha respondido inmunológicamente frente a un patógeno debido a una vacunación o a una infección. Esta característica de las vacunas DIVA es una herramienta fundamental en los programas de erradicación ya que permiten campañas conjuntas de vacunación/diagnóstico y erradicación mediante sacrificio.

Podemos hablar de tres tipos de "nuevas vacunas", producidas gracias a los avances biotecnológicos. El primero de ellos es el de las vacunas atenuadas mediante ingeniería genética, como consecuencia de la eliminación del gen o genes virulentos de un patóge- no. Al ser una modificación genética, la vacuna puede utilizarse sin peligro de reversión. Un ejemplo es la vacuna de Salmonella para ovejas y aves. Otro caso es la vacuna atenuada del herpesvirus de la enfermedad de Aujeszky mediante la eliminación de la glicoproteína gE que afecta a la virulencia del virus, pero no a su inmunogenicidad. Para aumentar más la seguridad de esta vacuna, se eliminó además el gen tk (timidinakinasa). Es un ejemplo de vacuna con un ensayo DIVA asociado, ya que los animales infectados con el virus desarrollan Ac frente a la proteína gE, mientras que los vacunados no, que ha sido empleada a gran escala demostrando su eficacia en campañas de erradicación. La misma estrategia se ha utilizado en el caso de la rinotraqueitis infecciosa bovina (IBR).

Un segundo grupo lo forman las vacunas basadas en vectores vivos. En este caso se usa un agente no patógeno con capacidad replicativa, que es capaz de producir en el propio organismo la proteína vacunal y producir una respuesta inmune específica frente a ella. El genoma del vector tiene que ser bien conocido y, normalmente, se pueden insertar más de un gen extraño. El virus vaccinia, el canarypox, adenovirus o coronavirus son algunos de los vectores más utilizados. La primera vacuna de este tipo comercializada, se basaba en el virus canarypox como vector, en cuyo genoma se insertó el gen de la hemaglutinina del virus de Newcastle. Otro ejemplo es la vacuna frente a la peste porcina clásica (PPC) basada en la expresión de la glicoproteína E2 del virus, utilizando como vector el virus de Aujeszky con los genes gE y TK delecionados.

Un tercer tipo lo constituyen las vacunas basadas en proteínas recombinantes que pueden ser péptidos o proteínas completas. Son vacunas no infectivas, biosintéticas, que utilizan distintos sistemas de expresión como Escherichia coli o células de mamífero, aves, insecto, levaduras o plantas para producir el antígeno de interés. La posibilidad de obtener industrialmente proteínas activas ha sido de gran ayuda para el desarrollo y la estandarización de nuevas vacunas y ensayos de diagnóstico y, por lo tanto, para la lucha frente a muchas enfermedades.

La primera vacuna comercializada basada en una proteína recombinante fue la del virus de la Hepatitis $B$, producida en levaduras. Existen también ejemplos de vacunas de proteínas recombinantes para bacterias como es el caso de la vacuna para Actinobacillus pleri pneumoniae.

La expresión de proteínas en células de insecto a través del uso de baculovirus como vector, ha sido 
una de las plataformas más versátiles y utilizadas. Al tratarse de un sistema eucariótico las proteínas que se producen pueden tener modificaciones que les confieren un mayor parecido a la proteína nativa $y$, por tanto, hace que las proteínas sintetizadas sean inmunológicamente activas. Existen dos vacunas subunidad comercializadas frente al virus de la peste porcina clásica (PPC) que se basan en la expresión de la proteína E2 en el sistema de baculovirus. Estas vacunas permiten un diagnóstico DIVA y han sido utilizadas de forma generalizada. Otro ejemplo es la vacuna de circovirus porcino (PCV), basada en la expresión de la proteína de la cápsida viral. En el caso concreto de la expresión de proteínas que forman la cápsida de ciertos virus, se ha observado que se ensamblan dando lugar a estructuras tan similares a la de la cápsida del virus nativo, que es incluso imposible distinguirlas. Dichas estructuras se denominan VLPs, por las siglas en inglés "Virus Like Particles", y son muy inmunogénicas. La mayoría de las VLPs descritas se han producido en el sistema de baculovirus (Crisci, Bárcena y Montoya, 2012; Liu et al., 2012; Roy y Noad, 2008). Son muy seguras, puesto que no llevan ADN infectivo, y muy inmunogénicas ya que son prácticamente idénticas al virus. Además, son estructuras muy estables y pueden asociarse a ensayos DIVA. Hay descritas más de 100 VLPs de 35 familias virales distintas (Parvoviridae, Caliciviridae, Birnaviridae, Picornaviridae...) (Zeltins, 2013). En la Figura 1 se muestra una fotografía de microscopía electrónica de cápsidas recombinantes de parvovirus porcino.
Además, las VLPs son muy eficaces como sistemas para presentar al sistema inmune, regiones inmunogénicas (epítopos) de cualquier patógeno de interés. Las secuencias de ADN que codifican por dichos epítopos, se introducen dentro del gen de la proteína de la cápsida, expresándose a la vez. De esta forma, en cada VLP hay varias copias del epítopo, generándose una respuesta inmune frente a la cápsida y frente al epítopo. Existen numerosos trabajos que describen su capacidad protectora (Antonis et al., 2006; Rueda et al., 1999).

Finalmente, tenemos las vacunas de $A D N$. Se trata de moléculas de $A D N$ en las que se introduce el gen que codifica la proteína diana frente a las que se quiere inmunizar. Cuando se inyecta, el ADN Ilega al núcleo celular poniéndose en marcha la maquinaria para sintetizar la proteína que desencadenará la respuesta inmune. Uno de los riesgos de estas vacunas, si bien es bajo, es que el ADN pueda integrarse en el ADN de la célula y provoque la transformación celular. Actualmente existen al menos dos vacunas de ADN comercializadas en Sanidad Animal: una frente al virus del Oeste del Nilo (WNV), que afecta fundamentalmente a caballos y puede también infectar aves y humanos, que está basada en la proteína de la cápsida y otra frente al virus de la necrosis hematopoyética infecciosa del salmón (IHN).

Debido a las ventajas que conllevan la utilización de las vacunas recombinantes, cabe pensar que cada vez se usarán más en Sanidad Animal. Sin embargo,

Figura 1. VLPs formadas por la proteína VP2 de la cápsida de Parvovirus (Fotografía de microscopía electrónica)

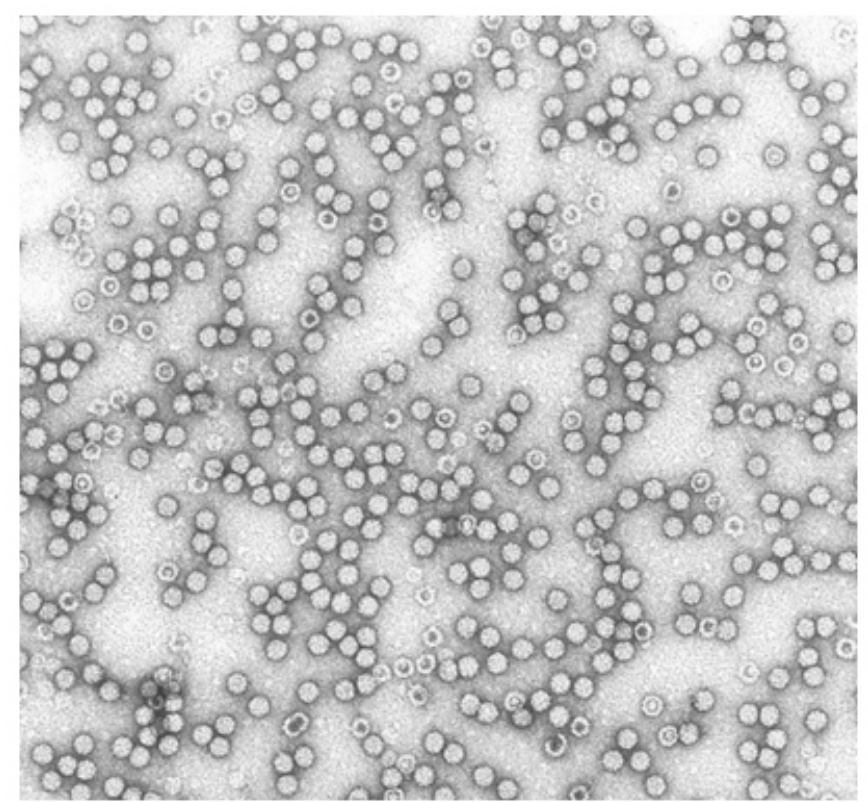


a pesar de ofrecer mayor seguridad que las vacunas convencionales, tienen que cumplir requerimientos muy estrictos para su aprobación que dificultan su comercialización.

A pesar de los avances biotecnológicos, hay enfermedades para las que no se ha conseguido ninguna vacuna eficaz, y por tanto, la única forma de control es el diagnóstico y el sacrificio de los animales infectados. Cuanto antes se detecte la enfermedad y más sensible y específico sea el diagnóstico, mejor será el control de la misma. Por otra parte, en el caso de enfermedades endémicas menos relevantes, en donde solo se ven afectados los índices productivos de las explotaciones, el seguimiento serológico de esas patologías constituye una herramienta clave en la gestión sanitaria de las mismas, permitiendo establecer pautas de manejo y programas de vacunación para mantener un equilibrio patógeno-hospedador que afecte lo menos posible a los parámetros productivos.

Tanto los servicios de sanidad oficiales de cada Estado, como los veterinarios y gestores sanitarios de las explotaciones pecuarias, demandan continuamente sistemas de diagnóstico más rápidos, eficaces y seguros. Esto promueve la constante mejora de los procesos diagnósticos veterinarios y, de nuevo aquí, la biotecnología vuelve a ser clara protagonista.

\section{BIOTECNOLOGÍA EN EL DIAGNÓSTICO DE ENFERME- DADES INFECCIOSAS}

La biotecnología aporta nuevas herramientas diagnósticas de gran utilidad sobre todo en aquellos casos en los que los microorganismos no pueden cultivarse, ya que permiten identificarlos sin necesidad de aislarlos. El diagnóstico puede hacerse de forma directa a través de la detección del agente infeccioso mediante técnicas inmunológicas o moleculares, o indirectamente, a través de la detección de anticuerpos específicos frente al patógeno (inmunidad humoral) o de citoquinas (inmunidad celular).

\section{Inmunoensayos y sus aplicaciones}

Los inmunoensayos se utilizan para detectar proteínas específicas de agentes patógenos, o respuestas de anticuerpos contra dichas proteínas. Se basan en interacciones inmunitarias antígeno-anticuerpo ( $\mathrm{Ag}-\mathrm{Ac}$ ) y por ello la producción de antígenos recombinantes mediante técnicas de ingeniería genética, así como la de anticuerpos monoclonales han supuesto una importante mejora cualitativa que ha permitido obtener unos índices de sensibilidad y especificidad hasta ese momento impensables. Los antígenos más adecuados son las proteínas en estado natural, puesto que proporcionan epítopos estructurales nativos, sin embargo, tienen que obtenerse a partir de cultivos celulares, son complicados de producir y caros, con periodos de validez cortos y pueden presentar problemas de infectividad por una inactivación incompleta. En la actualidad, se utilizan con frecuencia proteínas recombinantes las cuales ofrecen muchas ventajas respecto a los antígenos aislados de otras fuentes biológicas, como son: pureza, una gran actividad específica y estandarización entre lotes. Al igual que en el caso de las vacunas, los antígenos recombinantes se pueden obtener a partir de distintos sistemas ( $E$. coli, levaduras, células de insecto, etc.). Recientemente, el uso de plantas transgénicas para expresar proteínas de patógenos animales ha demostrado ser un método eficaz para la producción de grandes cantidades de proteínas recombinantes evitando el uso de materiales de origen animal como suero bovino fetal o huevos.

Con respecto a la producción de anticuerpos monoclonales (AcMs), la vía clásica de obtención se basa en la inmortalización de células productoras de anticuerpos (hibridomas) mediante fusión celular para producir un anticuerpo de una clase, especificidad y afinidad determinada (Koehler y Milstein, 1975). Una vez obtenidos estos hibridomas, se asegura el suministro indefinido, homogéneo y estable del AcM que producen, con sus características de especificidad y afinidad intactas. Esto supone una gran ventaja en la estandarización de métodos de diagnóstico que usan AcM.

Actualmente, las nuevas tecnologías permiten obtener anticuerpos específicos sin la inmunización de animales. La técnica "Phage display" se está utilizando para seleccionar péptidos, proteínas o anticuerpos en colecciones de fagos en cuya superficie exponen estos elementos (Smith y Petrenko, 1997). Como alternativa a la utilización de AcM, existen a su vez los Aptámeros (Tuerk y Gold, 1990; Ellington y Szostak, 1990). Se trata de cadenas sencillas de oligonucleótidos sintéti$\cos (A D N$ o ARN) identificados a través de un proceso combinatorial denominado SELEX (Systematic Evolution of Ligands by EXponential enrichment; cf. Stoltenburg, Reinemann y Strehlitz, 2007). Estas secuencias presentan una alta afinidad y especificidad por la diana de interés (antígeno viral, bacteria, etc.) mostrando características similares a los anticuerpos pero con mayor resistencia y estabilidad. Las herramientas diagnósticas basadas en aptámeros posiblemente serán de gran interés en el futuro para desarrollo de biosensores, etc. 
El límite para diferenciar entre inmunoensayos "clásicos", y aquellos "basados en biotecnología" no está claramente definido. Los inmunoensayos convencionales o clásicos se han basado en la detección de anticuerpos frente a un patógeno de interés utilizando, entre otras, las técnicas de neutralización viral en cultivos celulares, fijación de complemento, inmunodifusión en gel de agar o ensayos inmunoenzimáticos (ELISA). Los ensayos ELISA han sido una de las grandes aportaciones al diagnóstico inmunológico de las enfermedades infecciosas. En una muestra determinada se puede evaluar tanto la presencia de un patógeno como de anticuerpos frente a él. Posee innumerables aplicaciones en sanidad animal y, hoy en día, continúa siendo una de las técnicas más utilizadas en todas sus versiones: ELISA indirecto, de competición y de doble reconocimiento (DR), para detección de anticuerpos específicos en una muestra, y ELISA tipo sándwich para la detección de antígeno. Ejemplos de estos inmunoensayos son el ELISA de detección de Anaplasma (Trueblood, McGuire y Palmer, 1991), del virus de la Diarrea Viral Bovina (Mignon et al., 1991) y del virus de la Peste Bovina y del virus de la Peste de los Pequeños Rumiantes (Libeau et al. 1994) para detección del patógeno. Respecto a los ELISAs de detección de anticuerpos, han tenido gran importancia los ensayos DIVA utilizados en la erradicación del virus de la Pseudorabia en porcino (Ma et al., 2008) o en la erradicación de la Influenza Aviar de baja patogenicidad en aves domésticas (Marangon et al., 2003) entre otros.

Los "nuevos" inmunoensayos, además de mejorar la sensibilidad y la especificidad del diagnóstico, tienen dos objetivos principales:

En primer lugar, la detección múltiple de analitos (antígenos o anticuerpos) en un solo ensayo utilizando poco volumen de muestra. Un ejemplo de esta aproximación es la tecnología denominada Luminex, que implica la inmovilización de diferentes moléculas (antígenos o anticuerpos) en microesferas de poliestireno marcadas con distintas proporciones de dos colorantes fluorescentes sensibles a luz infrarroja (existen hasta la fecha 100 tipos de microesferas). Con esta técnica es posible analizar distintos parámetros en una muestra y en un solo pocillo, identificando cada uno de ellos de manera independiente. En el campo de la sanidad animal, se está trabajando en el desarrollo de ensayos para la detección simultánea de distintos patógenos entéricos o para detección y diferenciación de anticuerpos específicos frente a distintos serotipos de un virus o virus de la misma familia. Otra aproximación son los denominados micromatrices o microarrays, cuya superficie (vidrio, propileno...) está recubierta con analitos perfectamente ordenados con los que interaccionan las proteínas de la muestra. Un equipo especial, permite localizar con quién y con qué intensidad ha tenido lugar esa interacción. Inicialmente se desarrolló para detección de material genético, pero puede ser utilizada para la detección de proteínas y anticuerpos. Actualmente se utilizan con fines de investigación, pero es previsible que en un futuro próximo puedan llegar a ser de gran utilidad en diagnóstico.

En segundo lugar, hoy en día son cada vez más demandadas las técnicas diagnósticas que puedan realizarse fácilmente y en el sitio donde se localiza el problema (granja, clínica, mataderos, etc.). Es lo que se conoce como ensayos "Portable, Field, On-site, Pen-side o Point-of-care". El objetivo es, que se puedan tomar decisiones rápidas, que reduzcan los costes de diagnósticos más complejos y la diseminación de la enfermedad. Son fundamentales en países poco desarrollados, donde el transporte de las muestras a los laboratorios puede suponer mucho tiempo. La técnica inmunológica más ampliamente utilizada para este propósito es la inmunocromatografía (Figura 2). Está basada en el transporte de reactivos por capilaridad a través de una membrana porosa que permite detectar el complejo antígeno-anticuerpo de manera visual sin necesidad de equipo alguno proporcionando sensibilidad y rapidez en el diagnóstico. Son dispositivos de fácil manejo, con formato sencillo, muy estables, no necesitan refrigeración y son económicos. Se aplican en la detección de virus, bacterias, parásitos, hormonas y anticuerpos específicos de diferentes patologías. En la actualidad existen una gran variedad de ensayos comercializados para animales de compañía y de granja, y están siendo cada vez más demandados por los veterinarios.

\section{Diagnóstico Molecular}

Como se ha mencionado anteriormente, para que la gestión y control de las enfermedades infecciosas resulte eficiente, la identificación del patógeno debe ser rápida, sensible y específica. El desarrollo de las técnicas que analizan los ácidos nucleicos, supuso un gran avance en los últimos años del siglo XX. En particular, las técnicas basadas en la Reacción en Cadena de la Polimerasa (PCR) han revolucionado el diagnóstico de las enfermedades infecciosas. Sirven para amplificar y detectar de forma específica las moléculas del material genético (ADN o ARN) de un patógeno presente en una muestra. Son sensibles, específicas, rápidas y robustas y, además permiten su robotización y automatización, lo que facilita el análisis de un número elevado de muestras. Los usos de la técnica son muy variados, en sanidad animal la PCR ha sido muy utili- 
Figura 2. (A) Diagrama esquemático de un ensayo de inmunocromatografía. (B) Fotografía del resultado de un ensayo inmunocromatográfico para una (simplex) o dos patologías (dúplex)

(A)

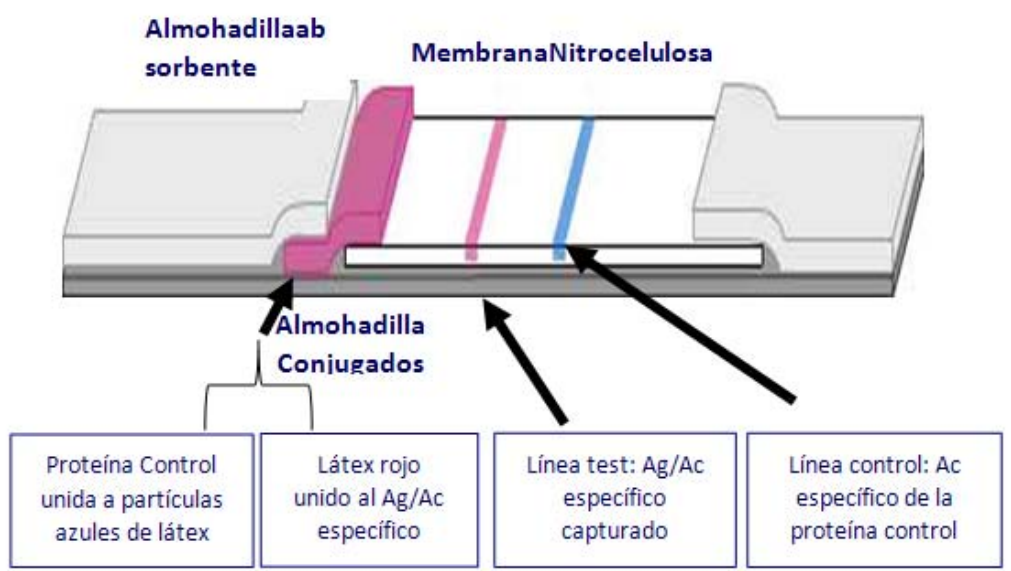

(B)

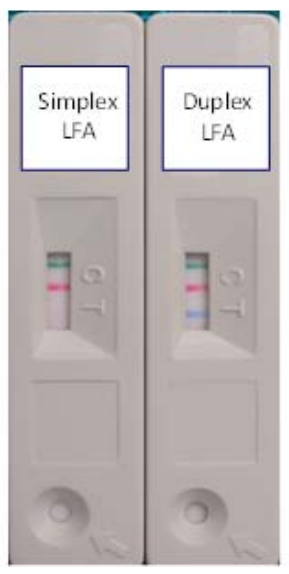

zada para la detección de agentes patógenos de difícil aislamiento o crecimiento lento, como los Mycoplasmas aviares (Hess, Neubauer y Hackl, 2007), y en aquellos casos de infecciones crónicas o persistentes en los que no hay serología y los animales infectados son reservorio del patógeno, como es el caso del virus de la diarrea viral bovina (BVD) (Lanyon et al., 2014).

En los últimos años, la técnica de PCR ha evolucionado notablemente. Hoy en día es posible la detección simultánea de distintos patógenos en una muestra, utilizando lo que se denomina $P C R$ múltiple. Es muy útil en el estudio y diferenciación de organismos relacionados, ejemplo Brucella (López-Goñi et al., 2011; López-Goñi et al., 2008), o en la diferenciación entre animales vacunados e infectados en planes de erradicación, por ejemplo en los casos de la Enfermedad de Auzjesky (ADV), la rinotraqueitis infecciosa o IBR (Mahajan et al., 2013; Schynts et al., 1999) o la Peste Porcina Clásica. La combinación de la PCR y su análisis posterior con enzimas de restricción (PCR-RFLP) o la amplificación al azar de secuencias repetidas en el genoma (RAPDS) permiten el análisis y diferenciación de aislados virales, herramienta clave a la hora de llevar a cabo estudios epidemiológicos, determinar la virulencia de un virus o para tomar una decisión con respecto al tipo de vacuna que se debe utilizar. En combinación con el análisis del material amplificado mediante electroforesis de campo pulsátil, permite también establecer patrones de diferenciación en infecciones bacterianas (Salmonellas, shigelas, cam- pylobacter). Actualmente, la $P C R$ en tiempo real o $P C R$ cuantitativa ( $q P C R$ ) está siendo muy utilizada en el diagnóstico de enfermedades infecciosas. La gran ventaja es que sirve para amplificar y simultáneamente cuantificar de forma absoluta o relativa los niveles de ácido nucleico en una muestra (Figura 3). Se basa en los mismos principios que la PCR, pero en este caso la detección del fragmento amplificado se realiza mediante sustancias fluorescentes. Se denomina PCR cuantitativa porque permite conocer la cantidad de ADN o ARN específico que existe en la muestra original. Esta técnica está siendo de gran utilidad, sobre todo en aquellos casos en que la carga del patógeno tiene un significado clínico. Un ejemplo seria la infección por Leishmania en perros. Esta enfermedad no se cura y la única solución es mantener el parasito inactivo. Mediante qPCR se puede evaluar el estado del animal y proporciona información de la eficacia de las terapias administradas. La aparición en los últimos años de equipos portátiles de qPCR, está permitiendo el uso de esta técnica directamente en el sitio de recogida de muestra. Siendo de gran utilidad en aquellas situaciones que requieren una toma de decisión rápida, como puede ser el movimiento de animales. Una de las aplicaciones más difundidas de la qPCR es el estudio de los genes que se expresan en una situación determinada, es lo que se conoce con el nombre de transcriptómica. Se analiza la expresión de un gran número de genes a la vez y está siendo muy útil para definir el tipo de respuesta inmune del huésped frente a distintos patógenos (Liu et al., 2009). 
Figura 3. Equipo de qPCR y análisis de resultados

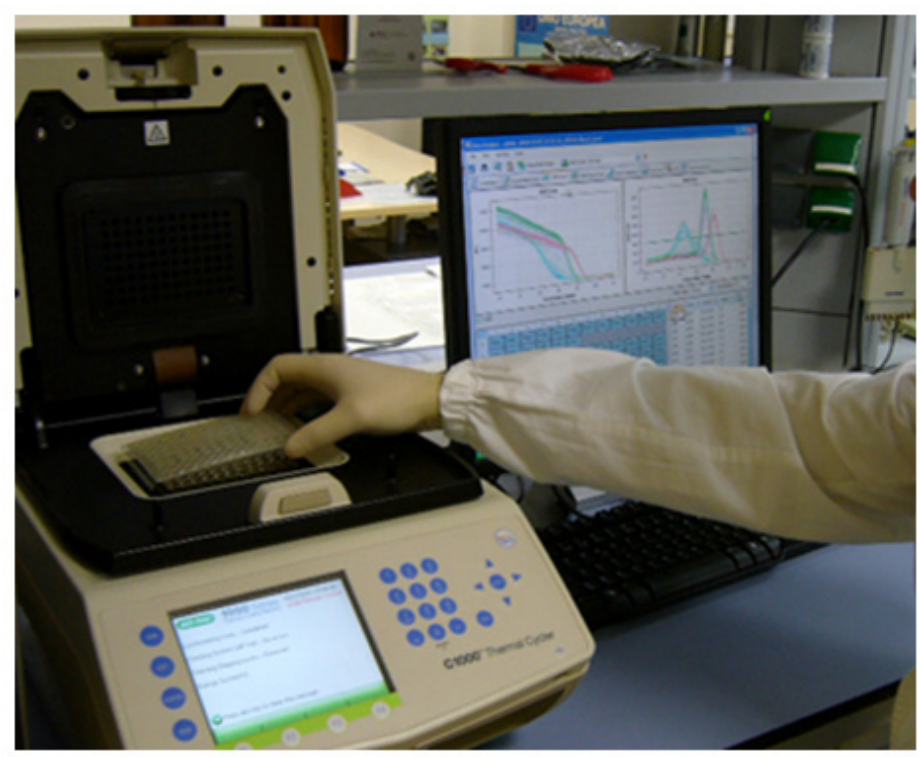

Actualmente, dado el interés de acercar las técnicas moleculares a laboratorios más sencillos, se ha desarrollado otra técnica alternativa a la PCR, denominada LAMP (del inglés, Loop mediated isothermal amplification), que se basa en la amplificación isotérmica del ácido nucleico. La reacción se realiza a temperatura constante y no se necesita ningún equipo sofisticado, además, el material amplificado puede detectarse de forma visual (James et al., 2010).

Al igual que en el caso del inmunodiagnóstico, las nuevas tendencias del diagnóstico molecular van dirigidas hacia el uso de métodos sencillos y eficaces que puedan ser realizados en el campo. Paralelamente, se están desarrollando equipos que integran módulos con los reactivos necesarios para realizar un diagnóstico automatizado, de forma que pueda llevarse a cabo en el campo y por personal no especializado.

Por otro lado, la secuenciación de las ácidos nucleicos permite conocer el genoma completo de un microorganismo, identificar nuevos patógenos, caracterizarlos, identificar patrones de resistencia a antimicrobianos, estudiar factores de virulencia, desarrollar nuevos métodos diagnósticos y vacunas... Es muy interesante la información que aporta la secuenciación a nivel epidemiológico, ya que la tipificación de los patógenos es la base de la Vigilancia Epidemiológica y da información de los patógenos y de su evolución. En los últimos años han avanzado enormemente los métodos robotizados de secuenciación masiva, unidos a los desarrollos bioinformáticos para la gestión de la información generada, lo que ha permitido aumentar el número, así como el tamaño, de secuencias que se pueden analizar. Con la aparición de estos métodos rápidos y de bajo coste, ha aparecido una nueva disciplina llamada Metagenómica. La Metagenómica consiste en la secuenciación de todo el ácido nucleico existente en una muestra determinada, sin necesidad de realizar aislamientos y cultivos. Los métodos de secuenciación masiva se están utilizando, entre otras aplicaciones, para la identificación de nuevos patógenos (Chiu, 2013) como el caso reciente del virus de Schmallenberg en ganado ovino (Goller et al., 2012), en el estudio de contaminaciones por agentes infecciosos, como la detección de Circovirus Porcino en una vacuna humana, y para el estudio de interacciones entre distintas poblaciones de microorganismos.

El uso de sondas marcadas con reactivos fluorescentes capaces de reconocer e hibridar con secuencias de ácidos nucleicos conocidas, permite el estudio complejo de patógenos en una muestra problema. EI desarrollo de micromatrices o "arrays o chips de ADN" permite ampliar el número de componentes de una mezcla que se puede analizar, llegándose a técnicas de análisis masivo. En sanidad animal esta técnica ha sido aplicada para análisis de patógenos entéricos y para la diferenciación de serotipos del virus de la lengua azul (BTV) y otros Orbivirus. El desarrollo de tecnologías basadas en chips hace tender a la miniaturización del formato analítico. Incluso miniaturizadas, estas plataformas detectan desde unos pocos, hasta miles de agentes patógenos en una sola muestra. Esta miniaturización está yendo acompañada de la miniaturización del equipo de laboratorio, permitiendo realizar análisis in situ y favoreciendo el uso en el campo de equipos sofisticados que hasta ahora estaban res- 
tringidos a los laboratorios. En definitiva, van a permitir un diagnóstico rápido y precoz de enfermedades infecciosas así como la rápida intervención de las autoridades competentes.

\section{Otras tecnologías}

Dado que la expresión de diferentes proteínas depende de factores fisiológicos y ambientales, la proteómica puede proporcionar una excelente visión general de los procesos de la enfermedad a nivel proteico. Muchos de los métodos que se utilizan en proteómica, como electroforesis en gel bidimensional (2DGE) y espectrometría de masas (MS) se establecieron hace muchos años, pero los avances de la secuenciación masiva, gracias en parte a la bioinformática, han revolucionado este campo. En el ámbito de la veterinaria, se están llevando a cabo proyectos de investigación, que producirán nuevas herramientas de diagnóstico para el futuro, obteniéndose mapas de proteoma de varios agentes patógenos importantes en veterinaria, como bacterias (Mujer et al., 2002), protozoos (Rout y Field, 2001) o nematodos (Yatsuda et al., 2003).

\section{MERCADO PRESENTE Y FUTURO DE LA BIOTECNOLOGIA APLICADA A LA SANIDAD ANIMAL}

La investigación en el campo de la biotecnología relacionada con la Sanidad Animal, ha dado lugar fundamentalmente a una serie de reactivos, ensayos de diagnóstico y vacunas, cuyos principales usuarios son las empresas de producción animal, clínicas veterina- rias y organismos públicos. Dentro del diagnóstico, los inmunoensayos y el diagnóstico molecular son las áreas donde más han influido los avances biotecnológicos. En la primera, los anticuerpos monoclonales han sido fundamentales para obtener nuevos ensayos más sensibles y específicos, y existen empresas en España que realizan la producción y distribución de los mismos. Pero ha sido el diagnóstico molecular donde más se han notado los grandes progresos biotecnológicos. Las distintas técnicas de amplificación, hibridación y secuenciación, han favorecido el incremento de nuevos ensayos y sus aplicaciones.

Por otra parte, en el sector de las vacunas también se observa un aumento paulatino del número de vacunas originadas a través de procesos biotecnológicos, si bien, el futuro es más incierto. La investigación y los ensayos necesarios previos, así como todos los procesos regulatorios que hay que pasar, antes de llegar con una vacuna al mercado, hace que muchas empresas no los puedan afrontar.

Según datos de Veterindustria del año 2012, en las grandes compañías farmacéuticas, el peso de los productos veterinarios apenas alcanza el $2 \%$ de sus facturaciones. Sin embargo, las estimaciones del volumen de negocio mundial de estos productos arrojan una cifra de 16.070.000.000 €. El volumen de los biológicos (vacunas y diagnósticos) utilizados en Sanidad Animal alcanza los 3.535.400.000 € (un $22 \%$ ). Los diagnósticos facturan anualmente 257.120.000 $€$, siendo el mercado español $12.850 .000 €$ (un $5 \%$ ) (Figura 4).

Figura 4. Mercado mundial y español de productos zoosanitarios y veterinarios

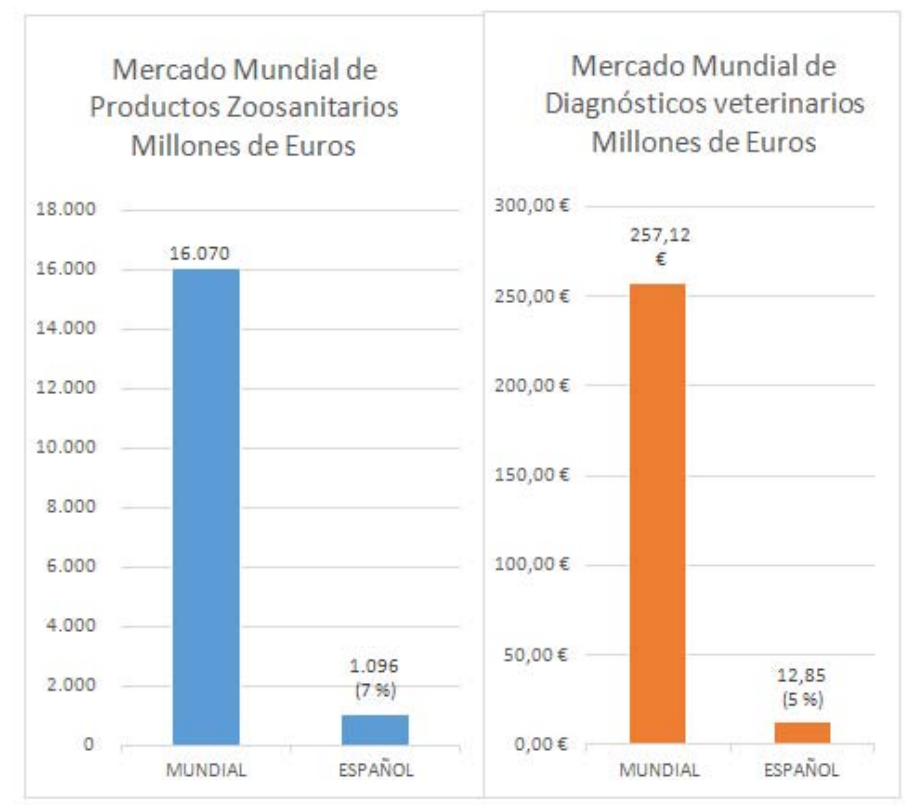


El crecimiento del mercado de biológicos es moderado, del orden de un $3 \%$ anual. Ello se debe a la caducidad de las patentes, sin que se produzca un número superior de nuevas patentes. En cuanto a los métodos diagnósticos, debería mantenerse de manera estable, pues el futuro habrá de basarse en la obtención de nuevas tecnologías que permitan analizar mayor número de parámetros por muestra, de forma rápida y manteniendo la máxima sensibilidad. Con ello se logrará el abaratamiento de los costes que permitan optimizar la producción y, consecuentemente, su utilización será más generalizada y tendrá consecuencias positivas en el bienestar animal.

La aplicación de los avances biotecnológicos en el sector de la sanidad animal tiene grandes perspectivas de crecimiento futuro. Para ello, la investigación y el posterior desarrollo tienen que ir de la mano y siempre teniendo en cuenta las demandas y necesidades del sector.

\section{BIBLIOGRAFÍA}

Antonis, A.F., Bruschke, C. J., Rueda, P., Maranga, L., Casas, J. I., Vela, C., Hilgers, L. A., Belt, P. B., Weerdmeester, K., Carrondo, M. J. y Langeveld, J. P.(2006). A novel recombinant virus-like particles vaccine for prevention of porcine parvovirus-induced reproductive failure. Vaccine, 24, pp. 5481-5490. http://dx.doi. org/10.1016/j.vaccine.2006.03.089

Barzon, L., Lavezzo, E., Costanzi, G., Franchin, E., Toppo, S. y Palù, G. (2013). Next-generation sequencing technologies in diagnostic virology. Journal of Clinical Virology, 58, pp. 346-350. http:// dx.doi.org/10.1016/j.jcv.2013.03.003

Brun, A., Bárcena, J., Blanco, E., Borrego, B., Dory, D., Escribano, J. M., Le Gall-Reculé, G., Ortego, J y Dixon, L. K.(2011).Current strategies for subunit and genetic viral veterinary vaccine development. Virus Research, 157, pp. 1-12. http://dx.doi. org/10.1016/j.virusres.2011.02.006

Chiu, C. Y. (2013). Viral pathogen discovery. Current Opinion in Microbiology, 16, pp. 468-478. http://dx.doi.org/10.1016/j. mib.2013.05.001

Crisci, E., Bárcena, J. y Montoya, M. (2012). Virus-like particles: the new frontier of vaccines for animal viral infections. Veterinary Immunology and Immunopathology, 148, pp. 211-225. http://dx.doi. org/10.1016/j.vetimm.2012.04.026

Ellington, A. y Szostak, J. (1990). In vitro selection of RNA molecules that bind specific ligands. Nature, 346, pp. 818-822. http://dx.doi.org/10.1038/346818a0

Goller, K. V., Hoper, D., Schirmeier, H., Mettenleiter, T. C. y Beer, M. (2012). Schmallenberg virus as possible ancestor of Shamonda virus. Emerging Infectious Diseases, 10, pp. 1644-1646.
Jackwood, M.W., Hickle, L., Kapil, S. y Silva, R. (2008). Vaccine development using recombinant DNA technology. Animal Agriculture's Future through Biotechnology, part 7. Council for Agricultural Science and Technology (CAST), no 38.

James, H. E., Ebert, K., McGonigle, R., Reid, S. M., Boonham, N., Tomlinson, J. A., Hutchings, G. H., Denyer, M., Oura, C. A., Dukes, J. P. y King, D. P. (2010). Detection of African swine fever virus by loopmediated isothermal amplification. Journal of Virological Methods, 164, pp. 68-74. http://dx.doi.org/10.1016/j. jviromet.2009.11.034

Hess, M., Neubauer, C. y Hackl, R. (2007). Interlaboratory comparison of ability to detect nucleic acid of Mycoplasma gallisepticum and Mycoplasma synoviae by polymerase chain reaction. Avian Pathology. Journal of the WVPA, 36, pp. 127-133. http://dx.doi. org/10.1080/03079450701203082

Köhler, G. y Milstein, C. (1975). Continuous cultures of fused cells secreting antibody of determined specificity. Nature, 256, pp. 495-497. http://dx.doi. org/10.1038/256495a0

Lanyon, S. R., Hill, F. I., Reichel, M. P. y Brownlie, J. (2014). Bovine viral diarrhoea: pathogenesis and diagnosis. The Veterinary Journal, 199, pp. 201-209. http://dx.doi.org/10.1016/j. tvjl.2013.07.024

Libeau, G., Diallo, A., Colas, F. y Guerre, L. (1994). Rapid differential diagnosis of rinderpest and pestes des petits ruminants using an immunocapture ELISA. Veterinary Record, 134, pp. 300-304. http://dx.doi.org/10.1136/ vr.134.12.300
Liu, C. H., Chaung, H. C., Chang, H. L., Peng, Y. T. y Chung, W. B. (2009). Expression of Toll-like receptor mRNA and cytokines in pigs infected with porcine reproductive and respiratory syndrome virus. Veterinary Microbiology, 136, pp. 266-276. http://dx.doi.org/10.1016/j. vetmic.2008.11.016

Liu, F., Ge, S., Li, L., Wu, X., Liu, Z. y Wang, Z. (2012). Virus-like particles: potential veterinary vaccine immunogens. Research in Veterinary Science, 93, pp. 553-559. http://dx.doi.org/10.1016/j. rvsc.2011.10.018

López-Goñi, I., García-Yoldi, D., Marín, C. M., Miguel, M. J. de, Barquero-Calvo, E., Guzmán-Verri, C., Albert, D. y GarinBastuji, B. (2011). New Bruce-ladder multiplex PCR assay for the biovar typing of Brucellasuis and the discrimination of Brucellasuis and Brucellacanis. Veterinary Microbiology, 154, pp. 152155. http://dx.doi.org/10.1016/j.vetmic.2011.06.035

López-Goñi, I., García-Yoldi, D., Marín, C. M., Miguel, M. J. de, Muñoz, P. M., Blasco, J. M., Jacques, I., Grayon, M., Cloekaert, A., Ferreira, A. C., Cardoso, R., Correa de Sá, M. I., Walravens, K., Albert, D. y Garin-Bastuji, B. (2008). Evaluation of a multiplex PCR assay (Bruce-ladder) for molecular typing of all Brucella species, including the vaccine strains. Journal of Clinical Microbiology, 46, pp. 3484-3487. http://dx.doi. org/10.1128/JCM.00837-08

Ma, W., Lager, K. M., Richt, J. A., Stoffregen, W. C., Zhou, F. y Yoon, K. J. (2008). Development of real-time polymerase chain reaction assays for rapid detection and differentiation of wild-type pseudora- 
bies and gene deleted vaccine viruses. Journal of Veterinary Diagnostic Investigation, 20, pp. 440-447. http://dx.doi. org/10.1177/104063870802000405

Mahajan, V., Banga, H. S., Deka, D., Filia, G. y Gupta, A. (2013). Comparison of diagnostic tests for diagnosis of infectious bovine rhinotracheitis in natural cases of bovine abortion. Journal of Comparative Pathology, 149, pp. 391-401. http:// dx.doi.org/10.1016/j.jcpa.2013.05.002

Marangon, S., Bortolotti, L., Capua, I., Bettio, M. y dalla Pozza, M. (2003). Low-pathogenicity avian influenza (LPAI) in Italy (2000-01): epidemiology and control. Avian Diseases, 47 (3 Suppl), pp. 10061009. http://dx.doi.org/10.1637/00052086-47.s3.1006

Marangon, S., Cecchinato, M. y Capua, I. (2008). Use of vaccination in avian influenza control and eradication. Zoonoses and Public Health, 55, pp. 6572. http://dx.doi.org/10.1111/j.18632378.2007.01086.x

Meeusen, E. N.T., Walker, J., Peters, A., Pastoret, P. P. y Jungersen, G. (2007). Current status of veterinary vaccines. Clinical Microbiology Reviews, 20, pp. 489-510. http://dx.doi.org/10.1128/ CMR.00005-07
Mignon, B., Dubuisson, J., Baranowski, E., Koromyslov, I., Ernst, E., Boulanger, D., Waxweiler, S. y Pastoret, P.-P. (1991). A monoclonal ELISA for bovine viral diarrhea pestivirus antigen detection in persistently infected cattle. Journal of Virological Methods, 35, pp. 177188. http://dx.doi.org/10.1016/01660934(91)90133-K

(2009). Biotechnology in the diagnosis of infectious diseases and vaccine development. En OIE Terrestrial Manual .

Rodríguez Ferri, E.F. (2012). Sanidad animal y nuevas tecnologías. Profesión Veterinaria, 77, pp. 61-98.

Roy P. y Noad, R. (2008). Virus-like particles as a vaccine delivery system: myths and facts. Human Vaccines, 4, pp. 5-12. http://dx.doi.org/10.4161/hv.4.1.5559

Rueda, P., Martínez-Torrecuadrada, J. L., Sarraseca, J., Sedlik, C., Barrio, M. del, Hurtado, A., Leclerc, C. y Casal, J. I. (1999). Engineering parvovirus-like particles for the induction of B-cell, CD4+ and $\mathrm{CTL}$ responses. Vaccine, 18, pp. 325-332. http://dx.doi.org/10.1016/ S0264-410X(99)00202-9

Schynts, F., Baranowski, E., Lemaire, M. y Thiry, E. (1999). A specific PCR to differentiate between $\mathrm{gE}$ negative vaccine and wildtype bovine herpesvirus type 1 strains. Veterinary Microbiology, 66, pp. 187-195 http://dx.doi.org/10.1016/ S0378-1135(99)00008-5

Smith, G. P. y Petrenko V. A. (1997). Phage Display. Chemical Review, 97, pp. 391-410. http://dx.doi.org/10.1021/ cr960065d

Stoltenburg, R., Reinemann, C., Strehlitz, B. (2007). SELEX-a(r)evolutionary method to generate high-affinity nucleic acid ligands. Biomolecular Engineering, 24, pp. 381-403. http://dx.doi. org/10.1016/j.bioeng.2007.06.001

Trueblood, E. S, McGuire, T. C. y Palmer, G. H. (1991). Detection of Anaplasma marginale rickettsemia prior to onset of clinical signs by using an antigen capture enzyme-linked immunosorbent assay. Journal of Clinical Microbiology, 29, p. 1542.

Tuerk, C. y Gold, L. (1990). Systematic evolution of ligands by exponential enrichment: RNA ligands to bacteriophage T4 DNA polymerase. Science, 249, pp. 505-510. http://dx.doi.org/10.1126/ science. 2200121

Zeltins, A. (2013). Construction and characterization of Virus-like Particles: a review. Molecular Biotecnology, 53, p. 92. 